\title{
Hybridizing Daphnia communities from ten neighbouring lakes: spatio-temporal dynamics, local processes, gene flow and invasiveness
}

Mingbo Yin ${ }^{1,2}$, Sabine Gießler ${ }^{2 *}$, Johanna Griebel ${ }^{2,3}$ and Justyna Wolinska ${ }^{2,3}$

\begin{abstract}
Background: In natural communities of cyclical parthenogens, rapid response to environmental change is enabled by switching between two reproduction modes. While long periods of asexual reproduction allow some clones to outcompete others, and may result in "clonal erosion", sexual reproduction restores genetic variation in such systems. Moreover, sexual reproduction may result in the formation of interspecific hybrids. These hybrids can then reach high abundances, through asexual clonal reproduction. In the present study, we explored genetic variation in water fleas of the genus Daphnia. The focus was on the short-term dynamics within several clonal assemblages from the hybridizing Daphnia longispina complex and the impact of gene flow at small spatial scales.

Results: Daphnia individuals belonged either to the parental species D. galeata and D. longispina, or to different hybrid classes, as identified by 15 microsatellite markers. The distribution and genotypic structure of parental species, but not hybrids, corresponded well with the geographical positions of the lakes. Within parental species, the genetic distance among populations of $D$. galeata was lower than among populations of D. longispina. Moreover, D. galeata dominance was associated with higher phosphorous load. Finally, there was no evidence for clonal erosion.
\end{abstract}

Conclusions: Our results suggest that the contemporary structure of hybridizing Daphnia communities from ten nearby lakes is influenced by colonization events from neighbouring habitats as well as by environmental factors. Unlike the parental species, however, there was little evidence for successful dispersal of hybrids, which seem to be produced locally. Finally, in contrast to temporary Daphnia populations, in which a decrease in clonal diversity was sometimes detectable over a single growing season, the high clonal diversity and lack of clonal erosion observed here might result from repeated hatching of sexually produced offspring. Overall, our study provides insights into spatio-temporal dynamics in a hybridizing Daphnia species complex in a recently established lake system, and relates genetic similarities of populations to a scenario of secondary invasion enhanced by environmental factors.

Keywords: Cyclical parthenogenesis, Daphnia longispina complex, Clonal richness, Microsatellite, Population structure

\section{Background}

In natural communities, cyclically parthenogenetic reproduction is a complex and adaptable reproductive strategy with strong implications for ecological and evolutionary processes, such as genetic diversity, clonal erosion, interspecific hybridization and gene flow. In this mode of reproduction, one or more generations of parthenogen-

\footnotetext{
* Correspondence: giessler@bio.Imu.de

${ }^{2}$ Department Biologie II, Evolutionsökologie, Ludwig-Maximilians-Universität, Großhaderner Str. 2, 82152 Planegg-Martinsried, Germany

Full list of author information is available at the end of the article
}

etically produced females alternate with a sexual generation formed by males producing sperm, and females producing haploid eggs. In cladoceran Daphnia, for example, females usually clone themselves by producing parthenogenetic daughters. Only when unfavourable conditions arise, such as food shortage, overcrowding, or change in temperature (e.g. [1]), do Daphnia individuals switch to the production of males and sexual haploid eggs that require fertilization, and then diapause. The general advantages of this reproductive mode are, on the one hand, enhanced genotypic diversity after sexual recombination 
and, on the other, high population growth rates during asexual periods (e.g. [2]).

The sexual phase of Daphnia's reproductive cycle results not only in the production of new genotypes, but also in the formation of interspecific hybrids if closely related species co-occur (e.g. [3]). Via asexual propagation, these hybrids can then sometimes reach high abundances (e.g. $[4,5])$. Such hybridization is especially common among the members of the Daphnia longispina complex and widespread among the species $D$. galeata and D. longispina (taxonomy revised in [6]). Most previous work on spatial patterns in the D. longispina complex explored Daphnia communities across large geographical regions (e.g. [5,7]). For example, Keller et al. [5] found that although $D$. galeata and D. longispina are present on both sides of the Alps, D. galeata is more common in lakes south of the Alps, whereas $D$. longispina dominates in the north. In addition to this apparent geographical gradient, differences in environmental conditions also play a role [5]. However, little is known about spatial variation in the hybridizing $D$. longispina complex over small scales, where dispersal among neighbouring habitats might be of great importance. The main stage for Daphnia's passive dispersal involves sexual diapause eggs being carried by water birds or transported by wind [8]. Since parental species produce more conspecific- than interspecific-crosses (therefore, more diapause eggs represent parental than hybrid genotypes), and since the "hatching success" of hybrid diapause eggs is inherently lower than of parental offspring $[9,10]$, parental species are expected to have higher gene flow capacities than hybrids [10].

In cyclical parthenogens, long periods of strict asexual reproduction might result in declines of clonal diversity. This process has been described in life history models as "clonal erosion" [11,12]. In the field, such a decrease in clonal diversity over the growing season has been reported for populations of Daphnia magna (e.g. [13]) and of cyclically parthenogenetic rotifers (e.g. [14]). With respect to Daphnia, evidence for clonal erosion comes from populations inhabiting temporary ponds (e.g. [13]). In these habitats, sexual and asexual periods are strictly separated, with sexually produced offspring hatching from diapause eggs mainly in spring, giving rise to a large number of clonal lineages. Due to subsequent clonal competition, genetic diversity decreases gradually. In contrast, previous studies of Daphnia populations from permanent lakes did not detect a consistent decrease in clonal diversity over a growing season (e.g. $[4,9])$. In these and many other studies, however, the "clones" were defined by few allozyme loci and might actually have represented clonal groups instead of single lineages [15]. This methodological constraint could bias the results in such a way that a potential decrease in genotypic diversity might have been undetectable. Indeed, clonal erosion might also shape the genetic structure in overwintering Daphnia populations, as argued in a study applying higher resolution microsatellite markers [16]. However, this conclusion remains speculative, because it was derived from an analysis of genotypic structure of Daphnia populations only at the end of the growing season. In a more detailed summer-autumn field survey of the $D$. longispina complex in two large lakes, clonal diversity was traced over time by genotyping Daphnia at 10 polymorphic microsatellite loci [17]. This set of markers is able to detect true clonal lineages [18], and the clonal diversity was found to be roughly constant [17]. In that study [17], the exclusion of the spring period, during which thousands of new genotypes are expected to hatch from diapause eggs, may have prevented the detection of an initial drop in diversity (e.g. $[13,19])$. Consequently, to rigorously test for clonal erosion in permanent habitats, a survey must start early in the year when genetic diversity is expected to be highest, and changes must be followed over the whole growing season until late autumn, otherwise a decline might remain undetected.

In the present study, we explored spatial patterns and temporal dynamics in the genotypic structure of Daphnia communities from several lakes inhabited by taxa of the $D$. longispina complex. This was undertaken over a small spatial scale, with study sites separated by $30 \mathrm{~km}$ at most. We applied high-resolution genotyping (15 microsatellite loci). Specifically, we tested if the relative frequencies of parental species and their hybrids correspond with the geographical proximity and/or environmental descriptors of the lakes. Then, we looked for changes in taxon composition between spring and autumn seasons. In addition, we estimated gene flow and compared genotypic similarities among populations belonging to either parental species or hybrids, in order to find evidence for invasiveness and/or local production of hybrid taxa. Finally, some lakes were tracked throughout one growing season to determine if clonal diversity decreases over time (i.e., clonal erosion).

\section{Results}

\section{Taxon assignment}

The 1934 Daphnia sampled from ten small lakes (flooded gravel pits) in and around Munich belonged to D. galeata, $D$. longispina or D. galeata $\times$ D. longispina hybrids. This was based on their position in the factorial correspondence analysis (FCA) in relation to the clusters formed by 49 well-defined reference genotypes representing three parental species or their respective hybrids (data not shown). To increase the resolution among genotypes, a separate FCA was run using reference genotypes from $D$. galeata, D. longispina and D. galeata $\times D$. longispina hybrids (35 genotypes). The FCA revealed that D. galeata originating from four lakes (FASA, FELD, HEIM and LERC; for abbreviation see Additional file 1: Table S1) 
clustered together. Meanwhile, D. longispina from OLCH formed a separate cluster in relation to $D$. longispina originating from four other lakes (LANG, LERC, LUSS and WALD; Figure 1). Taxon identity was further confirmed by NewHybrids [20], which assigned Daphnia genotypes to four of six possible classes: D. galeata $(47.9 \%)$, D. longispina (41.3\%), F1 (9.4\%) and F2 hybrids (0.8\%); no backcrosses were detected. Eleven individuals $(0.6 \%)$ could not be assigned, within a given $95 \%$ posterior probability, to any of the classes (Additional file 1: Table S1).

\section{Spatial patterns in taxon composition}

Across the sampling period (i.e. April - November 2011), only one parental species was present within a given lake, with the exception of FASA May sample $(98 \%$ D. galeata and $2 \% D$. longispina, $\mathrm{N}=48$ ) and LERC September sample $(80 \%$ D. galeata and $20 \%$ D. longispina; $\mathrm{N}=41$, Additional file 1: Table S1). These were, however, the single sampling dates when $D$. longispina was detected in these otherwise $D$. galeata-dominated communities. Notably, there was a significant relationship between geographical distance and differences in the spring taxon composition, when calculated for presence/absence data $(\mathrm{r}=0.23, P=0.044)$, but not for frequency data $(\mathrm{r}=0.18$, $P=0.084)$. Specifically, the lakes north and east of Munich were dominated by $D$. galeata or hybrids (FASA, FELD, FERI, LERC and HEIM), whereas the lakes north-west and west of Munich were dominated by $D$. longispina or hybrids (BOHM, LANG, LUSS, OLCH and WALD; Figure 2).

\section{Environmental preferences of parental taxa}

Two main principal components were extracted from six variables (physical and environmental characteristics; Additional file 2: Table S2) by eigenvalue $>1$. The first component PC1 (explaining 38.1\% of the variance) reflected mainly the trophic status and age of the lake; the higher the scores, the more eutrophic (i.e., high $\mathrm{P}_{\text {tot }}$ ) and older the lakes were. The second principal component $\mathrm{PC} 2$ (explaining $29.6 \%$ of the variance) was associated mainly with high lake depth and low nitrogen concentration (Figure 3). Overall, lakes inhabited by different Daphnia species were characterized by opposite component loadings on the first principal components analysis (PCA) axis. Thus, lakes inhabited by $D$. galeata were more eutrophic and older ( $\mathrm{df}=1, \mathrm{~F}=19.99, P=0.002$ ), while most lakes inhabited by $D$. longispina were of lower trophic level and younger (Figure 3). Moreover, when FASA and LERC were scored as lakes without $D$. longispina ( $D$. longispina was present at low frequencies; $0.3 \%$ ( 1 individual) and 2.5\% (8 individuals), respectively - all samples pooled), the difference in mean component loadings on the PC1 axis was significant for this species also $(\mathrm{F}=6.01, P=0.04$; otherwise: $\mathrm{F}=0.97$, $P=0.35$ ). In contrast, neither lake depth nor nitrogen concentration (loadings on PC2) had any influence on species

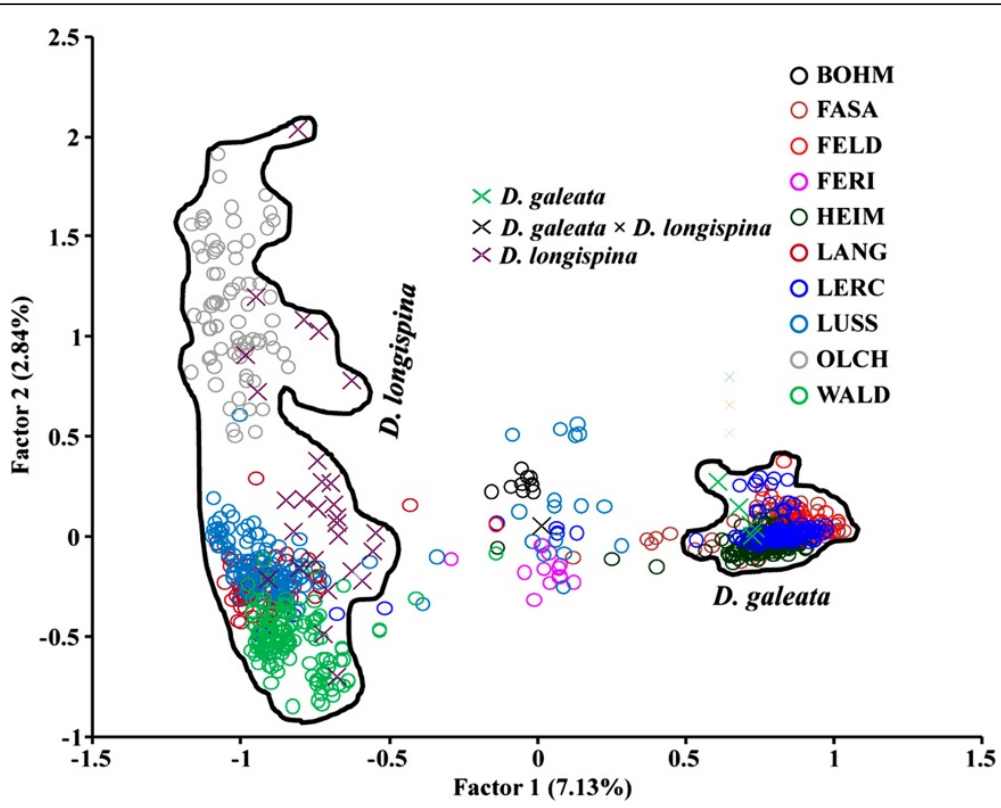

Figure 1 Factorial correspondence analysis displaying genetic similarities between individuals from the $D$. longispina complex sampled in 2011 from ten lakes in and around Munich, based on allelic variation at up to 15 microsatellite loci (data from monthly samples are pooled). For lake abbreviations see Additional file 1: Table S1. The 35 reference clones representing two parental species and their interspecific hybrids (indicated by crosses) are also shown (for a list of all reference clones see Table S1, Supporting information in Yin et al. 2010) [18]. The two outlines enclose the parental taxa as assigned by NewHybrids. 


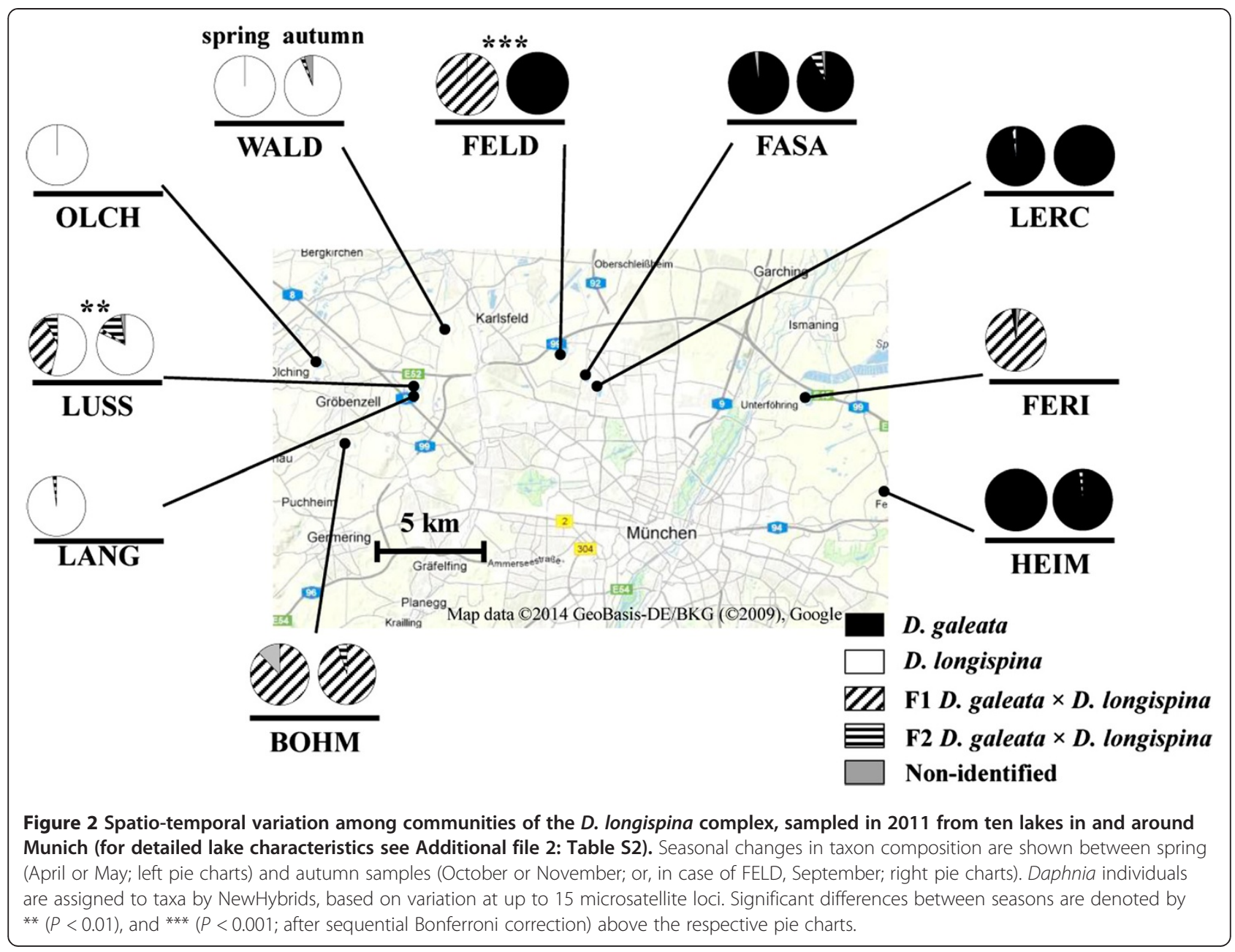

abundance $(D$. galeata: $\mathrm{F}=0.71, P=0.42 ; D$. longispina: $\mathrm{F}=0.54, P=0.35$; see Figure 3 ).

\section{Temporal changes in taxon composition}

Over time, Daphnia taxon composition changed significantly between spring and autumn in two of the seven lakes tested. In FELD, the community changed from complete dominance by F1 hybrids to complete dominance by $D$. galeata; in LUSS, $D$. longispina increased its abundance in comparison to the F1 hybrids (Figure 2).

\section{Genetic distances among spatially and temporally} isolated populations of parental species and hybrids

Similarities among populations from different lakes and sampling dates are illustrated by Unweighted Pair-Group Method with Arithmetic Mean (UPGMA) dendrograms based on Nei's genetic distance [21]. In general, populations from the same lake (although sampled in different months) clustered together (Figure 4). There were four exceptions to this pattern: D. galeata from LERC July sample, D. galeata from FASA April and May samples (Figure 4a), and D. longispina from WALD May sample
(Figure 4c). These particular samples were distinct from others from the respective lakes, because the genotypes which were dominant in these samples were not present in other periods (Additional file 3: Figure S1). For D. galeata and D. longispina, the genetic similarities among populations corresponded well with the geographical distribution of populations. Specifically, D. galeata from FASA, FELD and LERC (i.e., the lakes within a distance of $500 \mathrm{~m}$, see Figure 2) clustered together but were otherwise isolated from HEIM, a lake which is $15 \mathrm{~km}$ away (Figure 4a). D. longispina from LUSS and LANG (lakes only $\sim 100 \mathrm{~m}$ apart) clustered, but were isolated from D. longispina from LERC, WALD or OLCH, lakes separated by a distance of $>6 \mathrm{~km}$ (Figure 4c). In contrast, the clustering of hybrid populations did not correspond to their geographical position: hybrids from FERI and LUSS were the closest on the dendrogram, but the most isolated geographically (Figure 4b). Overall, the genetic distance between the most isolated populations within a given taxon was highest for hybrids $(\sim 0.55)$, half as great for $D$. longispina $(\sim 0.25)$, and one-tenth as great for D. galeata $(\sim 0.055$, Figure 4$)$. 


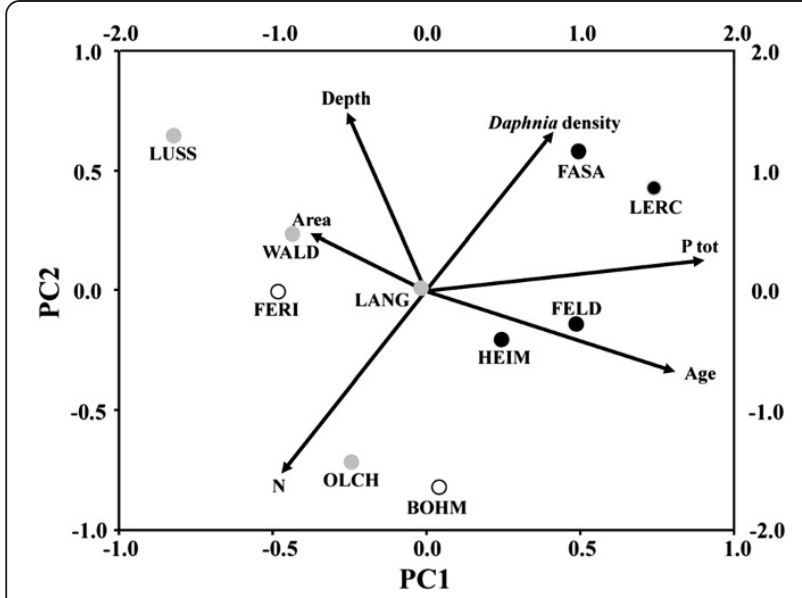

Figure 3 Association of Daphnia species distribution to mean Daphnia density and environmental descriptors of ten sampled lakes. The PCA plot displays (1) the projection of the original variables (see Additional file 2: Table S2) on to the first two components (coordinate scales: bottom and left) and (2) respective factorial loadings of lakes (coordinate scales: top and right). Arrows point to increasing importance for the respective variables. Each dot represents a lake. For lake abbreviations see Additional file 1: Table S1. Black dots: lakes inhabited by D. galeata; grey dots: lakes inhabited by D. longispina; white dots: neither parental species was detected (only hybrids were present). FASA and LERC (black dots with grey ring) are the only lakes where both species were detected. However, D. longispina was present at low frequencies: $0.3 \%$ (1 individual) and 2.5\% (8 individuals), respectively - all samples pooled.

\section{Gene flow and local production of hybrids}

The mean number of migrants among lakes (all pairwise comparisons per taxon) differed between the parental species and hybrids ( $\mathrm{df}=2, \mathrm{~F}=4.73, P=0.023$ ). Specifically, there were significantly more migrants among $D$. galeata populations $(\mathrm{Nm}=4.26, \mathrm{SD}=4.63, \mathrm{~N}=6)$ than among D. longispina $(\mathrm{Nm}=0.84, \mathrm{SD}=0.28, \mathrm{~N}=9)$ or hybrids $(\mathrm{Nm}=0.37, \mathrm{SD}=0.10, \mathrm{~N}=6)$, meaning that gene flow was largest for D. galeata (Post-Hoc test: $P<0.05$ ). Then, evidence for local production of hybrids was found. Specifically, the hybrids from LUSS (i.e. a lake where hybrids coexisted with parental species) were more likely assigned to the respective local parental $D$. longispina population than to populations of $D$. longispina from other lakes $(\mathrm{df}=17, \mathrm{t}=-14.2, P<0.001)$.

\section{Test for clonality}

Among 1856 individuals with complete multilocus genotypes (MLG) profiles (or data missing solely at the SwiD2 locus) 867 unique MLGs were detected. The hypothesis that individuals sharing identical MLGs were of sexual origin was rejected $(P<0.001$ in all cases), indicating that individuals with identical MLGs can be treated as the same clone.

\section{Temporal changes in clonal composition}

The most striking change was observed in FELD, where in May the community was dominated by only one hybrid clone. After the community crash, hybrids disappeared and multiple D. galeata clones were detected in September (Additional file 1: Table S1). Regarding the three populations for which it was possible to study changes in clonal composition in more detail (i.e., D. galeata from FASA and LERC, and D. longispina from WALD; samples available from April to November), a few common clones were present over a longer period of time, however the frequencies of these clones fluctuated greatly (Additional file 3: Figure S1). Furthermore, differences in clonal composition increased significantly with the time interval between sampling dates (i.e., indicating replacement of clones) for $D$. galeata from FASA (Figure 5a) and D. galeata from LERC (Figure 5b), but not for D. longispina from WALD (Figure 5c). There was no indication of clonal erosion in any of these three populations; genetic diversity (as measured by two indices: clonal richness $\mathrm{R}$ and the inverse of the Simpson index) did not decrease over time (Figure 6).

\section{Discussion}

In the present study, we surveyed spatio-temporal dynamics in hybridizing communities of Daphnia originating from ten neighbouring lakes. This is a first insight into genetic diversity, clonal erosion, hybridization and gene flow on such a small spatial scale which is tightly linked to the adaptable reproductive strategy in species complexes formed by cyclical parthenogens.

\section{Spatial pattern and importance of environmental factors on taxon composition}

A geographical separation was observed between communities dominated by $D$. galeata or D. longispina despite presumably ongoing dispersal between the neighbouring lakes. In a first step, lakes have been possibly colonized by extant neighbouring populations in the past. For example, Lußsee (LUSS) could have been colonized by D. longispina from Langwieder See (LANG), a lake created six decades earlier, and only $100 \mathrm{~m}$ away (see Figure 2). In Daphnia, and other zooplankton species, the advantage of first arrivals is relatively strong, due to their rapid population growth rate (e.g. [22,23]). Indeed, from studies of the colonization of artificial ponds, it has been reported that Daphnia populations were founded by only a few genotypes $[24,25]$. Moreover, after initial colonization, no new alleles were observed in these populations, indicating limited establishment success for genotypes arriving later [25]. However, it has also been observed that changes in environmental conditions might result in species shifts, especially in the D. longispina communities (e.g. [26]). Thus, independent from geographical proximity, 
(a)

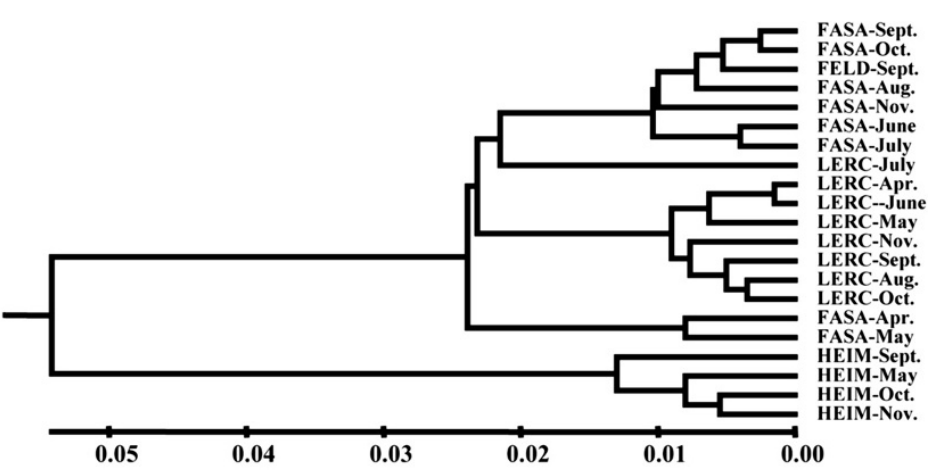

(b)

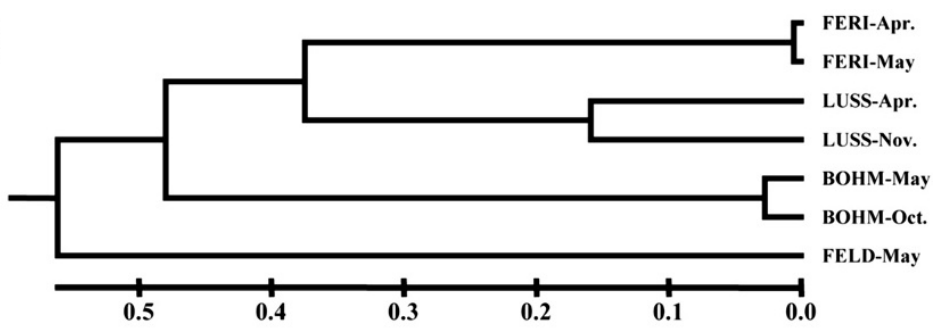

(c)

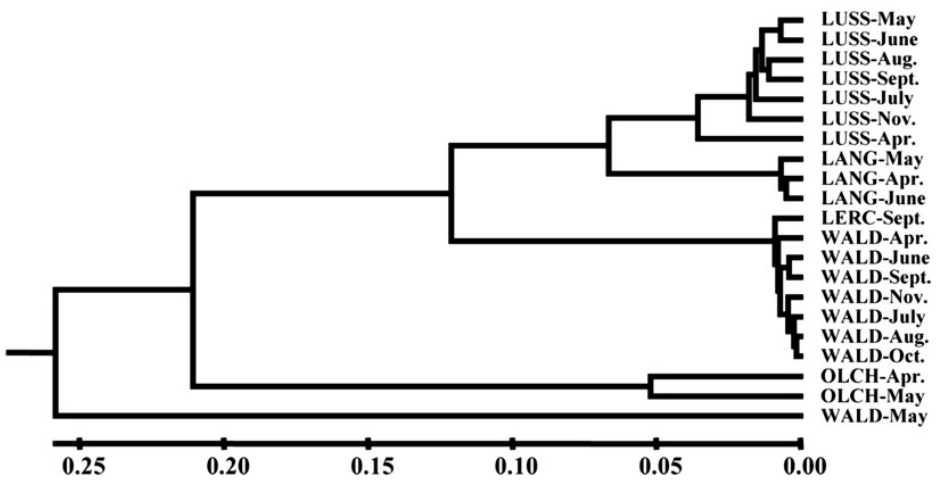

Figure 4 UPGMA clustering of Nei's genetic distances among populations of: (a) D. galeata, (b) hybrids (including F1 and F2 individuals) and (c) D. longispina, as sampled in $\mathbf{2 0 1 1}$ from ten lakes in and around Munich. Monthly samples are shown separately. The genetic distance was calculated based on variation at up to 15 microsatellite loci.

differences in lake environmental conditions may explain part of the observed spatial variation in taxon composition. Indeed, PCA and corresponding statistical tests showed that $D$. galeata occurred in more eutrophic (and older) habitats, and D. longispina in less eutrophic (and younger) ones. This pattern is consistent with a postulate of Flößner and Kraus [27] that D. galeata is promoted by eutrophication. Moreover, by comparing the physical parameters and water quality of several lakes, Keller et al. [5] concluded that $D$. longispina prefers habitats with low phosphorus load. Species-specific contrasting preferences were confirmed by the detection of a gradual replacement of $D$. longispina with $D$. galeata during periods of eutrophication [26], and by follow-up experimental tests showing differential fitness of these two parental species under various phosphorus loads [28].
Surprisingly, although D. galeata occurred exclusively in the oldest lakes, the genetic distance among populations of $D$. galeata was $\sim 1 / 5^{\text {th }}$ of that among populations of D. longispina, implying lower divergence time for D. galeata. This could possibly be explained by the fact that $D$. galeata spread later and, being more invasive and a better colonizer as suggested by the highest number of migrants, replaced former residents. Assuming that newly established artificial lake habitats are oligotrophic in the beginning and therefore first colonized by $D$. longispina, eutrophication might have allowed D. galeata to invade (see [26]). Overall, in our system the geographical separation between D. galeata and D. longispina dominated communities is consistent with the environmental conditions of the occupied habitats. Thus, geographical and environmental forces are not 


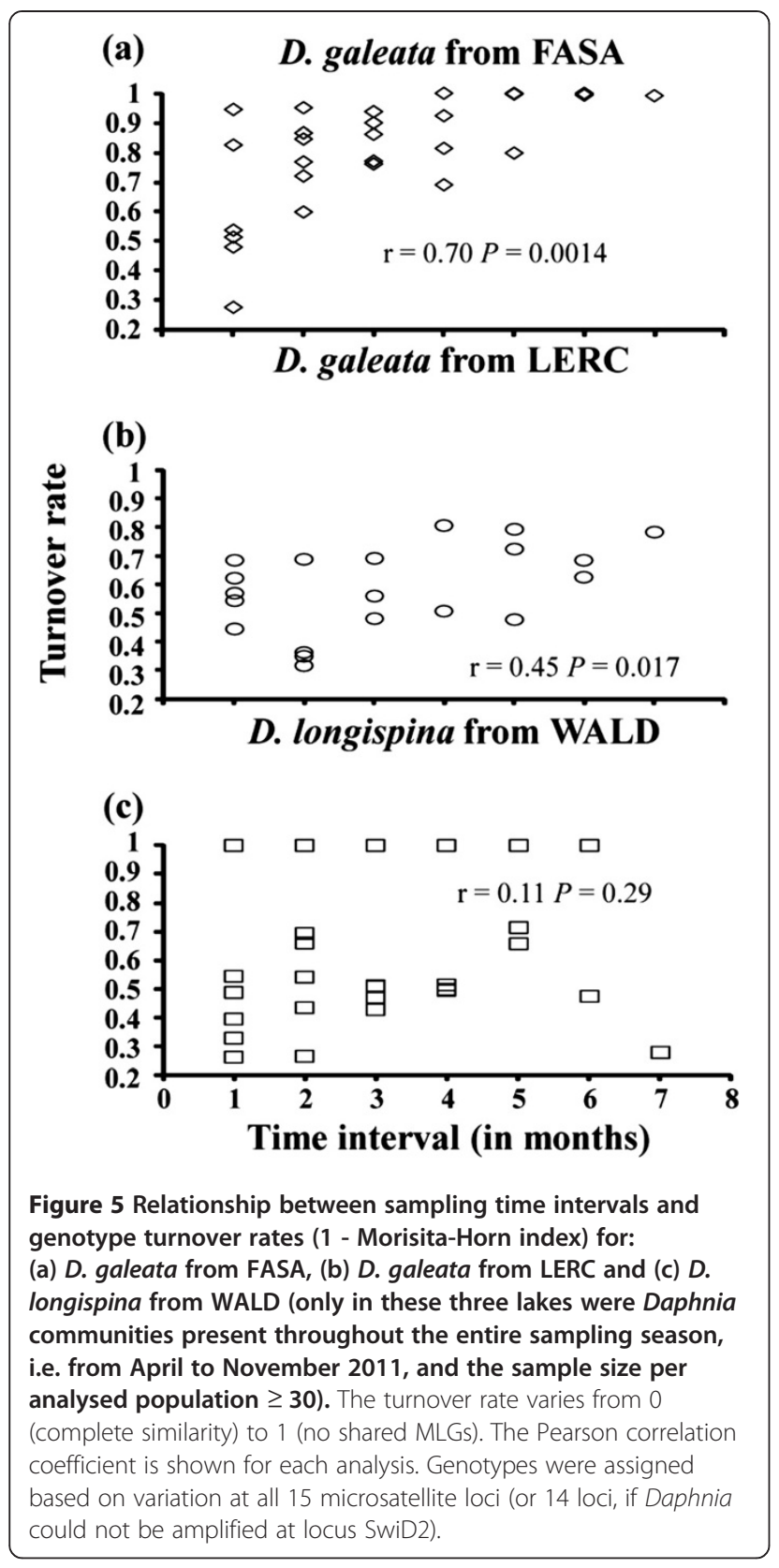

exclusive, and both most likely play a role in shaping Daphnia hybridizing communities.

\section{Temporal pattern and lack of clonal erosion}

The relative proportion of Daphnia taxa changed significantly between spring and autumn in two of the seven tested lakes. In addition, there was significant replacement of clones over time in two out of the three lakes for which a complete set of time series data was available. The observed changes may be due to the impact of environmental variation on the relative fitness of parental species and hybrids, as well as the fitness of specific clones within species, as has been shown in previous experimental surveys (e.g. [29-31]). However, although extinction of clones due to selection and random events should lead to an overall decrease in clonal diversity towards the end of the growing season [11], no signs of clonal erosion were detected in our study. Consistent with previous reports for permanent lakes (e.g. $[4,9]$ ), in our system the indices for clonal diversity remained roughly constant over the growing season, both for $D$. galeata and $D$. longispina populations (hybrids were not tested because of their small sample size). The presence of clonal erosion in Daphnia populations may thus depend on the habitat type $[11,32]$. Specifically, in contrast to temporary pond populations with clear separation of sexual and asexual phases in the Daphnia reproductive cycle (e.g. [33]), and for which the importance of clonal erosion has been documented (e.g. [13,34]), the two modes of reproduction often overlap in the larger permanent lakes (e.g. $[9,10,35])$. This can contribute to the maintenance of high clonal diversity throughout the year. Moreover, given that fitness of genotypes in the $D$. longispina complex varies strongly with environment (e.g. [30,31]), seasonal environmental changes may favour the maintenance of a high number of genotypes through fluctuating selection pressures.

\section{Local production of hybrids}

In contrast to parental species, neither the frequency of hybrids in the community, nor the clonal composition of hybrid populations was associated with the geographical neighbourhood of the lakes. Also, the UPGMA analysis showed that the highest genetic distance was detected among populations belonging to hybrids. Gene flow among hybrids was negligible and hybrids were more related to parents from the same lake than to parents from other lakes. These results complement evidence from previous studies showing that $D$. galeata $\times D$. longispina hybrids have impaired hatching success from diapause eggs $[9,10]$, possibly restricting their gene flow. This all suggests that these hybrids are rather locally produced, by multiple hybridization events [36].

\section{Conclusions}

In summary, by applying high-resolution microsatellite markers, we addressed spatial and temporal patterns in the hybridizing $D$. longispina complex on a small scale, in ten recently established lakes. We found that the distribution and genotypic structure of parental Daphnia species was consistent with the geographical position and the differences in environmental attributes of the lakes; in particular, the presence of D. galeata was related to higher phosphorous load. Furthermore, the genetic distance among populations of $D$. galeata was lower than among populations of $D$. longispina, implying lower divergence time for $D$. galeata. The explanation for this 
(a)

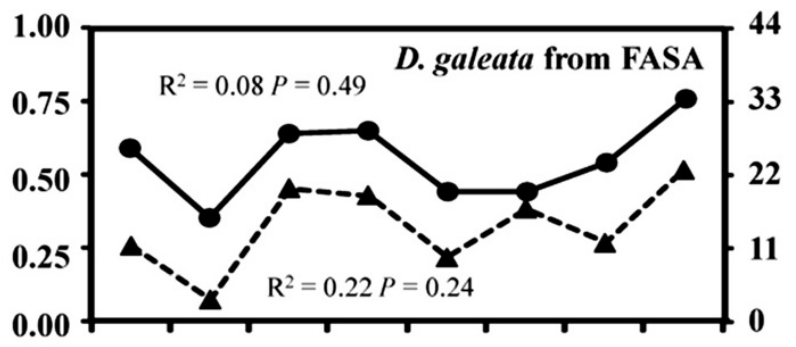

(b)
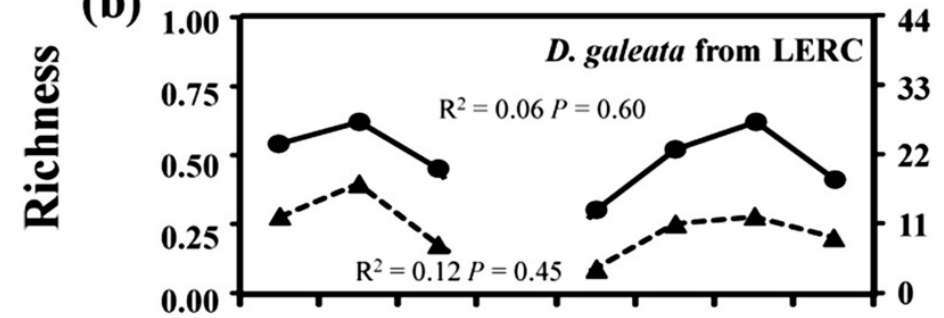

(c)

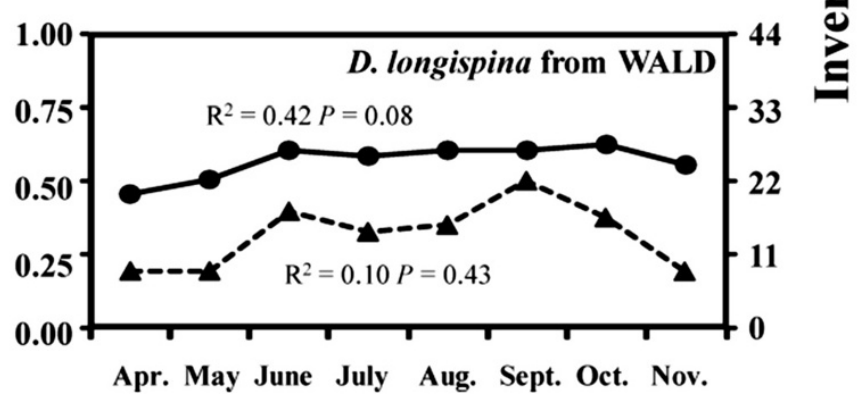

Figure 6 Temporal changes in clonal diversity, calculated as clonal richness R (solid line) or as the inverse Simpson index (dashed line) for (a) D. galeata from FASA, (b) D. galeata from LERC and (c) D. longispina from WALD (only in these three lakes were Daphnia communities present throughout the entire sampling season, i.e. from April to November 2011, and the sample size per analysed population $\mathbf{3 0}$ ). The upper/lower equation shows the results of linear regression analyses between time and clonal richness/inverse of the Simpson index, respectively. Missing data indicate a month when too few animals were available to calculate genetic indices of clonal diversity. The genetic indices were calculated based on variation at all 15 microsatellite loci (or 14 loci, if Daphnia could not be amplified at locus SwiD2).

may be that $D$. galeata spread later, being more invasive and a better colonizer, and then replaced former residents in lakes with high trophy level. Finally, no clonal erosion was detected across the studied lakes, suggesting that this phenomenon may depend on the habitat type and the extent of hatching of sexual offspring. Overall, the Daphnia system is a useful model to gain a better understanding of dynamics in hybridizing communities formed by cyclical parthenogens.

\section{Methods}

Ethics statement

Local administration has been contacted at the beginning of the study. Collection of zooplankton (Daphnia) did not require specific permissions as these samples were obtained from unprotected lakes that are open for public activities. Our study did not involve the use or collection of endangered or protected species.
Study sites and field sampling

Daphnia individuals were sampled from ten flooded gravel pits in and around Munich (Germany; Additional file 1: Table S1). The lakes were created between 1930 and 2000, most are small and shallow, and the maximum distance between them is about $30 \mathrm{~km}$. Geographical coordinates, lake age, lake size, maximum depth, total phosphorus concentration $\left(\mathrm{P}_{\text {tot }}\right)$, nitrogen concentration $(\mathrm{N})$ and Daphnia densities (averaged across monthly densities from April to November 2011) are given in the supplementary material (Additional file 2: Table S2). The phosphorus and nitrogen data were provided by Andreas Scholz from the Wasserwirtschaftsamt München (http://www.wwa-m.bayern.de/). These measurements were taken in spring, from the surface to the bottom of the lake at one-meter intervals (average $\mathrm{P}_{\text {tot }}$ and $\mathrm{N}$ values were then calculated). Zooplankton samples were collected monthly from April to November 
2011 (eight samples per lake; sampling interval was about 30 days). A $95-\mu \mathrm{m}$ plankton net was hauled through the whole water column at two different sites per lake within the deep basin (depth was measured using a portable depth sounder); the two samples were then pooled and preserved in 95\% ethanol. A random subsample was used for density counts (only adult females were taken into consideration). Then, ca. 50 adult females from the $D$. longispina complex were chosen randomly per sample for genotyping. Since Daphnia densities were sometimes very low, a complete time series of eight samples could be genotyped for only three lakes (FASA, LERC and WALD; for lake abbreviations see Additional file 1: Table S1). Two to seven samples were genotyped for the seven remaining lakes, resulting in 1934 Daphnia individuals being genotyped in total.

\section{Microsatellite genotyping}

The DNA of each individual Daphnia was extracted and genotyped at 15 microsatellite markers [37], in two multiplex polymerase chain reactions, following a protocol described elsewhere [18]. PCR products were analysed on an ABI PRISM 3700 capillary sequencer using a LIZ 500 labelled size standard. Genotypes were scored using GeneMapper version 3.7 (Applied Biosystems). Alleles at each locus were defined by their fragment length (in base pairs). Across different runs of genotyping, the consistency of alleles was checked, with locus-specific patterns of one reference genotype used in each run. This enabled us to adjust alleles with small differences in fragment length among different runs. In addition, there was no evidence of scoring errors due to stuttering, large allele dropout, or the presence of null alleles [38], as indicated by respective tests using MICRO-CHECKER 2.2.3 (10 ${ }^{4}$ permutations, [39]).

\section{Taxon assignment}

To display the genetic relatedness of multilocus genotypes (MLGs), factorial correspondence analysis (FCA) was applied in GENETIX 4.05 [40], on all unique MLGs together with 49 well-defined reference genotypes (same set of genotypes as in [18]), representing three parental species (D. cucullata, D. galeata, D. longispina) and their interspecific hybrids. It was confirmed that only D. galeata and $D$. longispina parental species were present in our dataset. Bayesian statistics were then used (in NewHybrids $1.1,[20])$ to assign individuals to one of six possible classes (i.e., two parental species, F1 and F2 hybrids and both backcrosses). The probability threshold for assignments was set to $95 \%\left(10^{6}\right.$ iterations after a burn-in of length $10^{6}$ ). All the following calculations of taxonspecific parameters are based on the taxon assignment in NewHybrids.

\section{Spatial patterns in taxon composition}

To test if the taxonomic similarity of Daphnia communities corresponds with the geographical proximity of the lakes, the correlation between pairwise Euclidean distances based on the taxon composition and respective pairwise geographical distances between sampling sites was evaluated using a Mantel test (5000 permutations, Past v2.07, [41]). Two estimates of Euclidean distances were derived in Past v2.07 for the spring samples (i.e., April or May), based on either the frequency, or the presence/absence, of five Daphnia groups. These groups included all classes as identified by NewHybrids (i.e., D. galeata, D. longispina, F1 hybrids and F2 hybrids; backcrosses were not detected) and an additional group of individuals that could not be identified by this method. All ten lakes were included in these tests.

\section{Environmental preferences of parental taxa}

A principal components analysis (PCA) of five environmental variables (i.e., lake age, lake size, maximum depth, total phosphorus concentration $\left(\mathrm{P}_{\text {tot }}\right)$, nitrogen concentration (N)) and mean Daphnia density (see Additional file 2: Table S2) was used to relate Daphnia species occurrence to combinations of these parameters. Variables that did not conform to normality were log-transformed. To test if Daphnia species were nonrandomly distributed along each component of the PCA, a one-way ANOVA was used with the presence/absence of each Daphnia species (i.e., D. galeata or D. longispina) as a main factor. The species presence/absence category was assigned based on the whole-year dataset (Additional file 1: Table S1). All ten lakes were included in these tests.

\section{Temporal changes in taxon composition}

The relative frequencies of five Daphnia groups (i.e., four classes: D. galeata, D. longispina, F1 hybrids and F2 hybrids identified by NewHybrids, and one group of individuals not identified by this method) were compared between spring (April or May) and autumn (October or November or, in the case of FELD, September), by applying a Monte Carlo approach with $10^{5}$ simulation runs [42]. Since separate tests were run for each of the seven lakes tested here (BOHM, FASA, FELD, HEIM, LERC, LUSS and WALD), sequential Bonferroni corrections [43] were applied when interpreting the results.

\section{Genetic distances among spatially and temporally} isolated populations of parental species and hybrids Pairwise Nei's genetic distances [21] were calculated in GENALEX 6 [44], among populations belonging to D. galeata, D. longispina or hybrids (F1 and F2 NewHybrids classes were pooled into one group of "hybrids", because of the low sample size of the F2 class). Population samples originating from different months were 
considered separately, and only populations with sample size $\mathrm{N} \geq 6$ were included. Then, genetic similarities among population samples were displayed using the Unweighted Pair-Group Method with Arithmetic Mean (UPGMA), as calculated in MEGA 4 [45].

\section{Gene flow and local production of hybrids}

The number of migrants between lakes was calculated per generation $(\mathrm{Nm})$ to estimate gene flow among populations of the two parental species (i.e., D. galeata and D. longispina) and the hybrids (F1 and F2 NewHybrids classes were pooled into one group of "hybrids", because of the low sample size of the F2 class), respectively (in GENETIX 4.05). The whole-year dataset was pooled per taxon and lake. For each of the three taxa, only lake samples with $\mathrm{N}>10$ were considered to represent a local population and included in this analysis. Then, one-way ANOVA and Post-Hoc Tests were used to test for taxon-specific differences in the number of migrants (in SPSS 20.0).

In addition, it has been tested if hybrids are more likely formed by the parental species inhabiting their home lake (i.e., local production of hybrids) than by the parental species from other lakes. Thus, for each individual hybrid genotype, the log-likelihood with which it was assigned to parental groups (specific parental species from the home lake versus pooled populations of the same parental species from the other lakes) was calculated in Arlequin 3.0 [46]. To distinguish statistical differences in respective log-likelihoods of hybrid assignments, a paired t-test $\left(10^{3}\right.$ bootstrap replications) was run in SPSS 20.0. Although hybrids and respective parental species co-existed in FELD and LUSS, the test above could only be performed for LUSS hybrids, because only one hybrid genotype was detected in FELD.

\section{Test for clonality}

To test the null hypothesis that an MLG encountered more than once was the result of sexual recombination, instead of clonal propagation, the $P_{\text {sex }}$ value was calculated in GENCLONE 2.0 [47]. The calculations were performed individually for each abundant taxon and lake. Only individuals characterized at all 15 microsatellite loci (or with missing data at a single locus, SwiD2; many $D$. longispina individuals could not be amplified at this locus) were included in these and the follow-up analyses of clonal structure (i.e., 1856 of 1934 genotyped Daphnia, see Additional file 1: Table S1).

\section{Temporal changes in clonal composition}

To test if differences in clonal composition increase with the time interval between samples, the clonal turnover rate was calculated between sampling dates (for all possible pairwise comparisons), per taxon and lake, by computing complements of the Morisita-Horn index (1-MH [48]; see also [49]) using the program SPADE [50] and $10^{4}$ bootstrap replications. This turnover rate takes into account not only changes in the presence of individual MLGs but also changes in MLG frequencies. Turnover rate varies from 0 (complete similarity) to 1 (no similarity). Then, the correlation between the pairwise turnover rate in clonal composition and the corresponding pairwise time differences between sampling dates (calculated in monthly intervals) was evaluated by a Mantel test (5000 permutations, Past v2.07). Finally, evidence was sought for the existence of an overall decrease in clonal diversity during the course of the growing season (i.e., clonal erosion). Clonal diversity was measured per taxon and sampling date as: (i) clonal richness $\mathrm{R}$, and (ii) the inverse of the Simpson index. Clonal richness $\mathrm{R}$ was calculated as $\mathrm{R}=(\mathrm{G}-1)$ / (N-1), where $\mathrm{G}$ is the number of genotypes and $\mathrm{N}$ represents sample size [51]. $\mathrm{R}$ varies from 0 (all individuals belong to one clone) to 1 (all individuals are different). The inverse of the Simpson index was calculated as $1 /\left(\sum \mathrm{P}_{\mathrm{i}}^{2}\right)$, where $\mathrm{P}_{\mathrm{i}}$ is the proportion of the $\mathrm{i}^{\text {th }}$ clone. This index thus includes additional information about the distribution of clones within samples. The minimum value of this index is 1 (all individuals belong to one clone) whereas the maximum value is the number of unique clones in the sample. Linear regression analyses were applied to test for the effect of time on clonal diversity (i.e., either clonal richness $\mathrm{R}$ or the inverse of the Simpson index) using R-software [52]. The tests were run only on those populations for which samples spanning the entire growing season were available: $D$. galeata from FASA, D. galeata from LERC and D. longispina from WALD (a minimum sample size was set of 30 individuals).

\section{Additional files}

Additional file 1: Table S1. Sample size and clonal diversity of Daphnia populations inhabiting ten lakes in and around Munich. Individuals were genotyped at 15 microsatellite loci.

Additional file 2: Table S2. Geographical location, physical and environmental characteristics of the ten lakes included in the present study.

Additional file 3: Figure S1. Temporal changes in clonal composition within populations of: (a) D. galeata from FASA, (b) D. galeata from LERC and (c) D. longispina from WALD (only in these three lakes were Daphnia communities present throughout the entire sampling season, i.e. from April to November 2011, and the sample size per analysed population $\geq 30$ ). The frequencies of the most common clones (i.e. frequency $\geq 10 \%$ in at least one sample) are indicated by different shading, whereas rare clones were pooled into one category (white area, up to 100\%). A blank square across the graph indicates a month when too few animals were available to calculate clonal frequencies. Clonal IDs were assigned based on variation at all 15 microsatellite loci (or 14 loci, if Daphnia could not be amplified at locus SwiD2).

\section{Competing interests}

The authors declare that they have no competing interests. 


\section{Authors' contributions}

SG and JW designed the study, MY carried out the molecular work and JG collected the environmental dataset. MY, SG and JW contributed to data analyses and preparation of the manuscript. All authors read and approved the final version.

\section{Acknowledgements}

We would like to thank Rita Jaenichen who organized and carried out the zooplankton sampling. Further, Rita Jaenichen and Sabine Radetzki helped with the genotyping whereas Monika Poxleitner counted the zooplankton samples for density estimates. Finally, we thank Andreas Scholz from Wasserwirtschaftsamt München who provided environmental data, and Thomas Hovestadt and two anonymous reviewers for comments on the manuscript and Mark Phillipo for the linguistic help. This research was funded by the German Science Foundation grant to JW (WO 1587/4-1) and the National Natural Science Foundation of China grant to MY (31200284).

\section{Author details}

'School of Life Science, Institute of Biodiversity Science, Fudan University, Handan Road 220, Shanghai, China. ${ }^{2}$ Department Biologie II, Evolutionsökologie, Ludwig-Maximilians-Universität, Großhaderner Str. 2, 82152 Planegg-Martinsried, Germany. ${ }^{3}$ Department of Ecosystem Research, Leibniz-Institute of Freshwater Ecology and Inland Fisheries,

Mueggelseedamm 301, 12587 Berlin, Germany.

\section{Received: 10 December 2013 Accepted: 4 April 2014}

Published: 12 April 2014

\section{References}

1. Pijanowska J, Stolpe G: Summer diapause in Daphnia as a reaction to the presence of fish. J Plankton Res 1996, 18(8):1407-1412.

2. Barton NH, Charlesworth B: Why sex and recombination? Science 1998, 281(5385):1986-1990.

3. Hebert PDN: Interspecific hybridization between cyclic parthenogens. Evolution 1985, 39:216-220.

4. Spaak P: Temporal changes in the genetic structure of the Daphnia species complex in Tjeukemeer, with evidence for backcrossing. Heredity 1996, 76:539-548.

5. Keller B, Wolinska J, Manca M, Spaak P: Spatial, environmental and anthropogenic effects on the taxon composition of hybridizing Daphnia. Phil Trans R Soc B 2008, 363(1505):2943-2952.

6. Petrusek A, Hobaek A, Nilssen JP, Skage M, Cerny M, Brede N, Schwenk K: A taxonomic reappraisal of the European Daphnia longispina complex (Crustacea, Cladocera, Anomopoda). Zool Scr 2008, 37(5):507-519.

7. Petrusek A, Seda J, Macháček J, Ruthová Š, Šmilauer P: Daphnia hybridization along ecological gradients in pelagic environments: the potential for the presence of hybrid zones in plankton. Phil Trans $R$ Soc $B$ 2008, 363(1505):2931-2941

8. Havel JE, Shurin JB: Mechanisms, effects, and scales of dispersal in freshwater zooplankton. Limnol Oceanogr 2004, 49(4):1229-1238.

9. Keller B, Spaak P: Nonrandom sexual reproduction and diapausing egg production in a Daphnia hybrid species complex. Limnol Oceanogr 2004, 49(4):1393-1400

10. Keller B, Wolinska J, Tellenbach C, Spaak P: Reproductive isolation keeps hybridizing Daphnia species distinct. Limnol Oceanogr 2007, 52(3):984-991.

11. de Meester L, Vanoverbeke J, de Gelas K, Ortells R, Spaak P: Genetic structure of cyclic parthenogenetic zooplankton populations - a conceptual framework. Arch Hydrobiol 2006, 167(1-4):217-244.

12. Vanoverbeke J, de Meester L: Clonal erosion and genetic drift in cyclical parthenogens - the interplay between neutral and selective processes. J Evol Biol 2010, 23(5):997-1012.

13. Carvalho GR, Crisp DJ: The clonal ecology of Daphnia magna. I. Temporal changes in the clonal structure of a natural population. J Anim Ecol 1987, 56:453-468.

14. Ortells R, Gomez A, Serra M: Effects of duration of the planktonic phase on rotifer genetic diversity. Arch Hydrobiol 2006, 167:203-216.

15. Thielsch A, Brede N, Petrusek A, de Meester L, Schwenk K: Contribution of cyclic parthenogenesis and colonization history to population structure in Daphnia. Mol Ecol 2009, 18(8):1616-1628.
16. Hamrova E, Mergeay J, Petrusek A: Strong differences in the clonal variation of two Daphnia species from mountain lakes affected by overwintering strategy. BMC Evol Biol 2011, 11:231.

17. Yin M, Petrusek A, Seda J, Wolinska J: Fine-scale temporal and spatial variation of taxon and clonal structure in the Daphnia longispina hybrid complex in heterogeneous environments. BMC Evol Biol 2012, 12:12.

18. Yin M, Wolinska J, Gießler S: Clonal diversity, clonal persistence and rapid taxon replacement in natural populations of species and hybrids of the Daphnia longispina complex. Mol Ecol 2010, 19(19):4168-4178.

19. Hairston NG, Hansen AM, Schaffner WR: The effect of diapause emergence on the seasonal dynamics of a zooplankton assemblage. Freshwat Biol 2000, 45(2):133-145.

20. Anderson EC, Thompson EA: A model-based method for identifying species hybrids using multilocus genetic data. Genetics 2002, 160(3):1217-1229.

21. Nei M: Estimation of average heterozygosity and genetic distance from a small number of individuals. Genetics 1978, 89(3):583-590.

22. de Meester L, Gomez A, Okamura B, Schwenk K: The monopolization hypothesis and the dispersal-gene flow paradox in aquatic organisms. Acta Oecol 2002, 23(3):121-135.

23. Mergeay J, de Meester L, Eggermont $H$, Verschuren D: Priority effects and species sorting in a long paleoecological record of repeated community assembly through time. Ecology 2011, 92(12):2267-2275.

24. Ortells R, Vanoverbeke J, Louette G, de Meester L: Colonization of Daphnia magna in a newly created pond: founder effects and secondary immigrants. Hydrobiologia 2014, 723(1):167-179.

25. Louette $G$, Vanoverbeke J, Ortells R, de Meester $L$ : The founding mothers: the genetic structure of newly established Daphnia populations. Oikos 2007, 116(5):728-741.

26. Brede N, Sandrock C, Straile D, Spaak P, Jankowski T, Streit B, Schwenk K: The impact of human-made ecological changes on the genetic architecture of Daphnia species. Proc Natl Acad Sci U S A 2009, 106(12):4758-4763.

27. Flößner D, Kraus K: On the taxonomy of the Daphnia hyalina-galeata complex (Crustacea: Cladocera). Hydrobiologia 1986, 137:97-115.

28. Spaak P, Fox J, Hairston NG Jr: Modes and mechanisms of a Daphnia invasion. P Roy Soc B-Biol Sci 2012, 279(1740):2936-2944.

29. Spaak P, Vanoverbeke J, Boersma M: Predator induced life history changes and the coexistence of five taxa in a Daphnia species complex. Oikos 2000, 89(1):164-174.

30. Wolinska J, Bittner K, Ebert D, Spaak P: The coexistence of hybrid and parental Daphnia: the role of parasites. P Roy Soc B-Biol Sci 2006, 273:1977-1983.

31. Seidendorf B, Boersma M, Schwenk K: Evolutionary stoichiometry: the role of food quality for clonal differentiation and hybrid maintenance in a Daphnia species complex. Limnol Oceanogr 2007, 52:385-394.

32. de Meester $L$ : Local genetic differentiation and adaptation in freshwater zooplankton populations: patterns and processes. Ecoscience 1996, 3(4):385-399.

33. Hebert PDN: Enzyme variability in natural populations of Daphnia magna. III. Genotypic frequencies in intermittent populations. Genetics 1974, 77:335-341.

34. Ruvinsky AO, Perelygin AA, Lobkov YI, Belyaev DK: Factors organizing and maintaining polymorphism in a cyclic parthenogenetic species Daphnia pulex. Heredity 1986, 57:15-22.

35. Hebert PDN: Genotypic characteristics of cyclic parthenogens and their obligately asexual derivates. In The Evolution of sex and its Consequences. Edited by Stearns S. Basel: Birkhäuser Verlag; 1987:175-195.

36. Spaak P: Hybridization in the Daphnia galeata complex: are hybrids locally produced? Hydrobiologia 1997, 360:127-133.

37. Brede N, Thielsch A, Sandrock C, Spaak P, Keller B, Streit B, Schwenk K Microsatellite markers for European Daphnia. Mol Ecol Notes 2006, 6(2):536-539.

38. Selkoe KA, Toonen RJ: Microsatellites for ecologists: a practical guide to using and evaluating microsatellite markers - appendix. Ecol Lett 2006, 9(5):615-629.

39. van Oosterhout C, Hutchinson WF, Wills DPM, Shipley P: MICRO-CHECKER software for identifying and correcting genotyping errors in microsatellite data. Mol Ecol Notes 2004, 4(3):535-538.

40. Belkhir K, Borsa P, Chikhi L, Raufaste N, Bonhomme F: GENETIX 4.05, logiciel sous Windows TM pour la génétique des populations. In Laboratoire Génome, Populations, Interactions, CNRS UMR 5000. Montpellier (France): 
1996-2004, Université de Montpellier II. Available from URL: http://www. univ-montp2.fr/.

41. Hammer $\varnothing$, Harper DAT, Ryan PD: PAST paleontological statistics software package for education and data analysis. Palaeontol Electroni 2001, 4:1-9.

42. Sham PC, Curtis D: Monte-Carlo tests for associations between disease and alleles at highly polymorphic loci. Ann Hum Genet 1995, 59:97-105.

43. Rice WR: Analyzing tables of statistical tests. Evolution 1989, 43:223-225.

44. Peakall R, Smouse PE: GENALEX 6: genetic analysis in Excel. Population genetic software for teaching and research. Mol Ecol Notes 2006, 6(1):288-295.

45. Tamura K, Dudley J, Nei M, Kumar S: MEGA4: Molecular evolutionary genetics analysis (MEGA) software version 4.0. Mol Biol Evol 2007, 24(8):1596-1599.

46. Excoffier L, Laval G, Schneider S: Arlequin (version 3.0): An integrated software package for population genetics data analysis. Evol Bioinform 2005, 1:47-50

47. Haond A, Belkhir K: GENCLONE: a computer program to analyse genotypic data, test for clonality and describe spatial clonal organization. Mol Ecol Notes 2007, 7(1):15-17.

48. Magurran AE: Ecological Diversity and its Measurement. Cambridge: University Press; 1988.

49. Seda J, Petrusek A, Machacek J, Smilauer P: Spatial distribution of the Daphnia longispina species complex and other planktonic crustaceans in the heterogeneous environment of canyon-shaped reservoirs. J Plankton Res 2007, 29(7):619-628.

50. Chao A, Chazdon RL, Colwell RK, Shen TJ: A new statistical approach for assessing similarity of species composition with incidence and abundance data. Ecol Lett 2005, 8(2):148-159.

51. Dorken ME, Eckert CG: Severely reduced sexual reproduction in northern populations of a clonal plant, Decodon verticillatus (Lythraceae). J Ecol 2001, 89(3):339-350

52. R Development Core Team: R: A Language and Environment for Statistical Computing. Vienna, Austria: R Foundation for Statistical Computing; 2009.

doi:10.1186/1471-2148-14-80

Cite this article as: Yin et al:: Hybridizing Daphnia communities from ten neighbouring lakes: spatio-temporal dynamics, local processes, gene flow and invasiveness. BMC Evolutionary Biology 2014 14:80.

\section{Submit your next manuscript to BioMed Central and take full advantage of:}

- Convenient online submission

- Thorough peer review

- No space constraints or color figure charges

- Immediate publication on acceptance

- Inclusion in PubMed, CAS, Scopus and Google Scholar

- Research which is freely available for redistribution

Submit your manuscript at www.biomedcentral.com/submit
(O) Biomed Central 